\title{
Desenvolvimento de uma rede neuro-fuzzy para predição da temperatura retal de frangos de corte
}

\author{
Leandro Ferreira ${ }^{1}$ \\ Tadayuki Yanagi Junior ${ }^{2}$ \\ Alison Zille Lopes ${ }^{3}$ \\ Wilian Soares Lacerda ${ }^{4}$
}

\begin{abstract}
Resumo: Objetivou-se com o presente trabalho desenvolver e validar uma rede neuro-fuzzy, baseada no algoritmo LOLIMOT, para a predição da temperatura retal de frangos de corte. A rede neuro-fuzzy foi desenvolvida no ambiente computacional SCILAB 4.1, com base em três variáveis de entrada: temperatura do ar, umidade relativa e velocidade do ar, tendo como variável de saída, a temperatura retal. Resultados experimentais mostraram que o desvio padrão médio entre os valores simulados e medidos foi de $0,1{ }^{\circ} \mathrm{C}$. A rede neuro-fuzzy se apresenta como um sistema inteligente híbrido satisfatório para a predição da temperatura retal de frangos de corte, agregando características da lógica fuzzy baseada na teoria dos conjuntos fuzzy, às redes neurais artificiais.
\end{abstract}

Palavras-chave: sistemas híbridos, modelos lineares locais, ambiente térmico

Abstract: The goal of this work was to develop and validate a neuro-fuzzy
intelligent system (LOLIMOT) for rectal temperature prediction of broiler chickens.
The neuro-fuzzy network was developed using SCILAB 4.1, on the ground of three

Departamento de Engenharia, Universidade Federal de Lavras (UFLA), Caixa Postal 3037, Lavras/MG, Brasil

${ }^{1}$ le.ferreira@gmail.com

${ }^{2}$ yanagiedeg.ufla.br

3 alisonzille@gmail.com 
input variables: air temperature, relative humidity and air velocity. The output variable was rectal temperature. Experimental results, used for validation, showed that the average standard deviation between simulated and measured values of RT was $0.11^{\circ} \mathrm{C}$. The neuro-fuzzy system presents as a satisfactory hybrid intelligent system for rectal temperature prediction of broiler chickens, which adds fuzzy logic features based on the fuzzy sets theory to artificial neural networks.

Key words: hybrid systems, local linear models, thermal environment

\section{Introdução}

Em busca de um ambiente adequado para criação de frangos de corte em galpões climatizados, sistemas de controle são utilizados para monitorarem variáveis climáticas e concentração de gases, e controlar sistemas de ventilação e nebulização [1]. De acordo com a prática de manejo adotada e o ambiente térmico, que caracteriza toda a fase de produção, o animal pode se encontrar em conforto ou desconforto. Quando o ambiente se torna desconfortável, o animal intensifica os mecanismos de conservação ou dissipação de calor, com o objetivo de manter a sua homeotermia [2].

Em condições de desconforto térmico, com temperatura e umidade elevadas, o animal terá dificuldade de perder calor, tendo como resultado, o aumento da temperatura corporal [3]. Com o aumento da temperatura corporal, em função do aumento da temperatura do ar, o animal eleva a frequência respiratória e reduz o consumo de ração, na tentativa de manter a temperatura corporal dentro de limites fisiológicos [4]. Para avaliar a temperatura corporal de frangos de corte, que oscila em torno de $41^{\circ} \mathrm{C}$, utiliza-se a temperatura retal, que representa adequadamente o estado de conforto ou desconforto animal diante do ambiente térmico.

Avaliando o ambiente de criação, as variáveis ambientais influenciam o conforto do animal e consequentemente, a produção. Assim, um sistema inteligente pode ser utilizado para avaliar o ambiente térmico no interior de galpões, levando em consideração respostas produtivas ou fisiológicas.

Sistemas inteligentes baseados na lógica fuzzy são sistemas capazes de trabalhar com informações imprecisas e transformá-las em uma linguagem matemática de fácil implementação computacional. A lógica fuzzy é uma ferramenta interessante na tomada de decisão, possibilitando, assim, o controle do ambiente térmico no interior de instalações avícolas, modelando incertezas por meio do emprego de conjuntos fuzzy. A teoria dos conjuntos fuzzy foi estudada primeiramente por Lofti Zadeh, na Universidade de Califórnia, em 1965 [5]. Os conjuntos fuzzy representam as informações em função de termos

\footnotetext{
${ }^{4}$ lacerda@ufla.br
} 
linguísticos, expressando conceitos vagos, assim como a linguagem natural [6]. De acordo com [7], um sistema inteligente fuzzy é constituído pelas etapas de fuzzificação, inferência, base de regras e defuzzificação. A base de regras, que relaciona as variáveis de entrada do sistema com as de saída, é construída de acordo com opiniões de especialistas, apresentando dessa maneira, caráter subjetivo.

Mesmo com o processamento do conhecimento de forma clara, sistemas inteligentes fuzzy apresentam limitações quanto à elaboração da base de regras, pois dependendo da quantidade de variáveis a serem modeladas com os seus respectivos conjuntos fuzzy, a construção do sistema se torna inviável.

As redes neurais artificiais, baseadas no sistema nervoso biológico, são modelos computacionais de processamento de informação que consistem em um grande número de unidades computacionais simples, chamadas de neurônios. Cada neurônio processa os sinais de entrada da rede, os quais são controlados por pesos sinápticos que se adaptam, durante o treinamento, por um algoritmo de aprendizado [8]. De acordo com [9], redes neurais artificiais é uma geração nova e promissora de sistema de processamento de informação, as quais demonstram habilidade para aprender, reaprender e generalizar a partir de dados e padrões treinados A possibilidade de uma maior aproximação entre os sistemas artificiais e os sistemas nervosos biológicos, constitui uma das principais motivações para o desenvolvimento de neurônios lógicos fuzzy [10].

Além da área de ambiência animal $[11,12,13,14]$, sistemas inteligentes utilizando a teoria dos conjuntos fuzzy e redes neurais artificiais têm sido desenvolvidos, principalmente, na área de ciências da saúde $[15,16,17]$.

Como alternativa a modelagem de sistemas, surgem os sistemas híbridos, com a finalidade de trabalhar limitações contidas em cada técnica, como por exemplo, à construção subjetiva da base de regras num sistema fuzzy. Dessa maneira, sistemas híbridos neuro-fuzzy são propostos utilizando algoritmos como, ANFIS (Adaptive Network-based Fuzzy Inference System) e LOLIMOT (Local Linear Model Tree), onde se tem o raciocínio impreciso, caracterizado pela lógica fuzzy, e a capacidade de aprendizado, caracterizada pelas redes neurais artificiais $[18,19,20]$.

Uma rede neuro-fuzzy, baseada no algoritmo LOLIMOT [21], é caracterizada pela aproximação de um modelo não linear por uma série de modelos lineares, com aprendizado baseado em árvores. $\mathrm{O}$ espaço de entrada é dividido em partições onde se têm os modelos lineares locais. Em cada partição tem-se uma função de pertinência fuzzy, também denominada de função peso. Os modelos lineares e suas funções peso normalizadas são apresentados na camada intermediária da rede, sendo que, a solução final da rede neuro-fuzzy é obtida por meio do somatório da solução de cada modelo linear [22, 23]. A Figura 1 ilustra a estrutura de uma rede neuro-fuzzy (LOLIMOT), com p entradas, m neurônios, com suas funções de validação $\Phi_{\mathrm{m}}$ e resultados $\mathrm{y}_{\mathrm{m}}$, e saída y. 


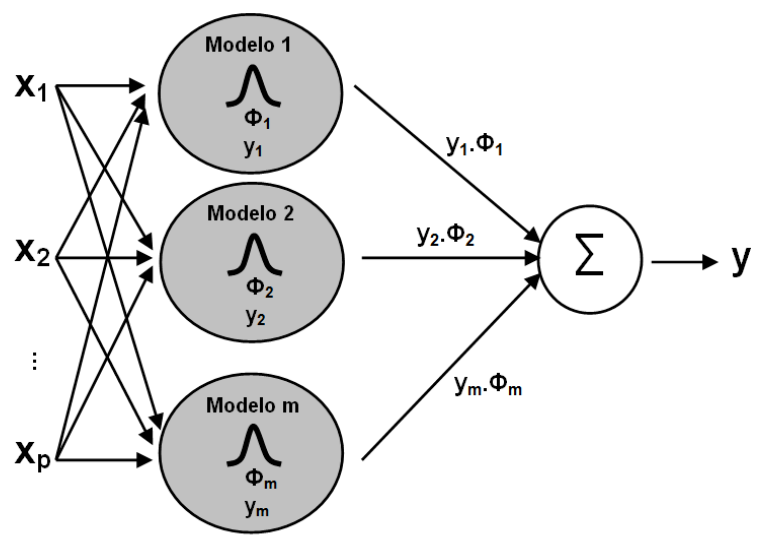

FIGURA 1. Estrutura de uma rede neuro-fuzzy (LOLIMOT).

Devido à dificuldade existente em elaborar uma base de regras considerando variáveis ambientais para a predição de respostas fisiológicas, objetivou-se com a presente pesquisa, desenvolver uma rede neuro-fuzzy, baseada no algoritmo LOLIMOT, por ser facilmente implementada num ambiente computacional gratuito disponível na internet, para predizer a temperatura retal de frangos de corte e, consequentemente, avaliar o ambiente de produção.

\section{Material e métodos}

\subsection{Desenvolvimento da rede neuro-fuzzy}

Para a construção da rede neuro-fuzzy foram utilizadas como variáveis de entrada: temperatura do ar $\left(T,{ }^{\circ} \mathrm{C}\right)$, umidade relativa $(U R, \%)$ e a velocidade do ar $\left(V, \mathrm{~m} \mathrm{~s}^{-1}\right)$. Com base nas variáveis de entrada, a rede prediz a temperatura retal $\left(T R,{ }^{\circ} \mathrm{C}\right)$ de frangos de corte. Foram utilizados 230 dados médios experimentais baseados nas pesquisas desenvolvidas por [24, 25, 26, 27], coletados em câmaras climáticas em diferentes fases de produção, sendo que 161 dados foram utilizados para o treinamento da rede e 69 para a validação. A Tabela 1 apresenta a estatística descritiva dos 230 dados médios experimentais utilizados.

TABELA 1. Estatística descritiva dos dados médios experimentais.

\begin{tabular}{ccrcc}
\hline & $\boldsymbol{T}\left(\mathbf{C}^{\mathbf{0}} \mathbf{C}\right)$ & $\boldsymbol{U R}(\boldsymbol{\%})$ & $\boldsymbol{V}\left(\mathbf{m ~ s}^{\mathbf{- 1}}\right)$ & $\left.\boldsymbol{T R}_{\text {média }}{ }^{\mathbf{0}} \mathbf{C}\right)$ \\
\hline Média & 28,02 & 60,90 & 1,90 & 41,83 \\
Mediana & 26,30 & 63,00 & 2,00 & 41,73 \\
Desvio padrão & 5,65 & 16,33 & 1,17 & 0,80 \\
Mínimo & 16,00 & 20,00 & 0,00 & 40,87 \\
Máximo & 36,00 & 90,00 & 4,00 & 43,90 \\
\hline
\end{tabular}


O treinamento da rede neuro-fuzzy foi realizado com a validação, onde o treinamento é baseado no critério de convergência em caso de aumento do residual, que é a soma do quadrado dos desvios do conjunto de validação. Para tanto, utilizou-se o toolbox LOLIMOT 1.0 inserida no software gratuito SCILAB 4.1 (disponível em http://www.scilab.org/communities/developer_zone/scilab_versions/oldreleases/scilab_4.1), considerando o número de partições do espaço de entrada igual a 10, originando 10 modelos lineares, e funções peso tipo gaussiana com desvio padrão de 0,33 , sendo estes valores sugeridos por [21].

Para a construção da rede neuro-fuzzy foi considerado como valor de saída para cada modelo linear $y_{i}$, o resultado da equação (1).

$$
y_{i}=\omega_{i 0}+\omega_{i 1} x_{1}+\omega_{i 2} x_{2}+\omega_{i 3} x_{3}
$$

Os parâmetros de cada modelo linear foram estimados pela técnica de mínimos quadrados ponderados através das funções peso. Para tanto, considerou-se uma matriz de dados $X$, na qual cada coluna representou uma medida $X^{T}$ no instante $k\left(X_{k}^{T}\right)$, uma matriz diagonal de pesos $Q_{i}$, na qual cada entrada $q_{k}$ foi o valor da função peso para um correspondente $x_{k}$, e as saídas desejadas $\mathrm{y}$, obtendo, desta forma, os parâmetros $\omega_{i}$ do iésimo modelo, de acordo com a equação (2) [23].

$$
\omega_{i}=\left(x^{T} Q_{i} X\right)^{-1} x^{T} Q_{i} y
$$

A função peso tipo gaussiana $\mu_{i}(x)$ para cada modelo linear, definida pela equação (3), apresentou dois parâmetros: o centro $C_{i j}$ e o desvio padrão $\sigma_{i j}$. Dessa maneira, a função peso foi normalizada conforme a equação (4).

$$
\begin{gathered}
\mu_{i}(x)=\exp \left(\frac{\left(x_{1}-c_{i 1}\right)^{2}}{-2 \sigma_{i 1}^{2}}\right) \cdot \exp \left(\frac{\left(x_{2}-c_{i 2}\right)^{2}}{-2 \sigma_{i 2}^{2}}\right) \cdot \exp \left(\frac{\left(x_{3}-c_{i 3}\right)^{2}}{-2 \sigma_{i 3}^{2}}\right) \\
\Phi_{i}(x)=\frac{\mu_{i}(x)}{\sum_{i=1}^{10} \mu_{i}(x)}
\end{gathered}
$$

Assim, a saída $y$ da rede neuro-fuzzy foi obtida pela equação (5). 


$$
y=\sum_{i=1}^{10}\left(\omega_{i 0}+\omega_{i 1} x_{1}+\omega_{i 2} x_{2}+\omega_{i 2} x_{3}\right) \cdot \Phi_{i}(x)
$$

Conforme o espaço de entrada proposto, ocorreu a partição através de hiperquadriláteros. De acordo com [23], o algoritmo LOLIMOT foi realizado da seguinte maneira:

1. obteve-se o primeiro hiper-quadrilátero que englobou todos os dados de entrada e estimou o modelo linear global $y=\omega_{0}+\omega_{1} x_{1}+\omega_{2} x_{2}+\omega_{3} x_{3}$.

2. para cada uma das $D$ dimensões $(j=1, \ldots, \mathrm{D})$ que o hiper-quadrilátero i possuiu, procedeu-se da seguinte maneira:

- cortou-se o hiper-quadrilátero i em duas metades ao longo da dimensão j;

- estimou-se o modelo linear local para cada metade;

- calculou-se o erro de aproximação global para este corte.

3. determinou-se qual o corte que apresentou o menor erro de aproximação.

4. considerou-se o corte com menor erro e ajustou-se uma função peso normalizada no centro de cada um dos novos hiper-quadriláteros formados através deste corte;

5. determinou-se os modelos locais para cada partição e o erro local.

6. escolheu-se a partição que obteve o maior erro local e realizou um novo corte.

7. caso o critério de convergência ainda não fosse atingido, retornava-se ao passo 2.

Fim.

\subsection{Simulações}

Para exemplificar o uso da rede neuro-fuzzy, procederam-se algumas simulações visando predizer a $T R$ no interior de galpões climatizados de frangos de corte, com o auxílio do programa computacional 'SisTúnel' desenvolvido por [28], utilizando como entrada, a temperatura do ar de $25,56{ }^{\circ} \mathrm{C}$ e umidade relativa de $69,2 \%$ do ambiente externo, além dos dados referentes a estrutura do galpão, com dimensão de 12 × 125 × 2,5 m, cobertura de telhas de cimento amianto, alvenarias do tipo tijolo furado, dez exaustores succionando o ar externo e, ventilação em modo túnel (pressão negativa) e sistema de resfriamento evaporativo dos tipos material poroso umedecido e nebulização. Desta forma, o programa computacional simulou o comportamento das variáveis do ambiente térmico no interior do galpão em 125 pontos (volume de controle), distanciados em $1 \mathrm{~m}$, ao longo do comprimento do galpão. Os frangos de corte estavam alojados na densidade de 14 aves $\mathrm{m}^{-2}$, com idade $37 \pm 1$ dia e peso médio de $2,40 \pm 0,13 \mathrm{~kg}$. 


\section{Resultados e discussão}

A Tabela 2 apresenta os modelos lineares obtidos pela simulação, na qual foram realizadas 10 partições no espaço de entrada, originando 10 modelos lineares, e os resíduos de treinamento referentes a cada modelo. A Tabela 3 apresenta os cortes inferiores e superiores referentes a cada partição. Pela Figura 2 pode-se visualizar as partições realizadas.

TABELA 2. Modelos lineares, considerando as partições do espaço de entrada.

\begin{tabular}{ccc}
\hline Partição & \multicolumn{1}{c}{ Modelo linear } & $\begin{array}{c}\text { Resíduo de } \\
\text { treinamento }\end{array}$ \\
\hline $\mathbf{1}$ & $T R=40,25255+0,03376 . T+0,00021 . U R+0,05395 . V$ & 0,48390 \\
$\mathbf{2}$ & $T R=36,65065+0,16960 . T+0,00048 . U R-0,02613 . V$ & 0,29848 \\
$\mathbf{3}$ & $T R=46,22647-0,15670 . T+0,01573 . U R+0,00002 . V$ & 0,42969 \\
$\mathbf{4}$ & $T R=38,68580+0,01633 . T+0,07387 . U R-0,26871 . V$ & 0,65616 \\
$\mathbf{5}$ & $T R=37,43153+0,18677 . T-0,00114 . U R-0,03308 . V$ & 0,00442 \\
$\mathbf{6}$ & $T R=20,82760+0,64407 . T+0,00805 . U R-0,05849 . V$ & 1,05348 \\
$\mathbf{7}$ & $T R=32,90497+0,29911 . T-0,01010 . U R-0,13923 . V$ & 1,19131 \\
$\mathbf{8}$ & $T R=50,71253-0,37014 . T-0,00013 . U R-0,02885 . V$ & 0,34299 \\
$\mathbf{9}$ & $T R=40,39839+0,02893 . T-0,00169 . U R+0,07685 . V$ & 0,47548 \\
$\mathbf{1 0}$ & $T R=47,35860-0,02700 . T-0,05784 . U R-0,02285 . V$ & 0,00516 \\
\hline
\end{tabular}

TABELA 3. Cortes inferiores e superiores referentes a cada partição do espaço de entrada.

\begin{tabular}{ccccccc}
\hline \multirow{2}{*}{ Partição } & \multicolumn{3}{c}{ Corte inferior } & \multicolumn{3}{c}{ Corte superior } \\
& $\boldsymbol{T}\left({ }^{\circ} \mathbf{C}\right)$ & UR $(\boldsymbol{\%})$ & $\boldsymbol{V}\left(\mathbf{m ~ s}^{-\mathbf{1}}\right)$ & $\boldsymbol{T}\left({ }^{\circ} \mathbf{C}\right)$ & $\boldsymbol{U} \boldsymbol{R}(\boldsymbol{\%})$ & $\boldsymbol{V}\left(\mathbf{m ~ s}^{-\mathbf{1}}\right)$ \\
\hline $\mathbf{1}$ & 16,0 & 20,0 & 0,0 & 21,0 & 90,0 & 2,0 \\
$\mathbf{2}$ & 26,0 & 20,0 & 0,0 & 31,0 & 90,0 & 4,0 \\
$\mathbf{3}$ & 31,0 & 20,0 & 0,0 & 33,5 & 55,0 & 4,0 \\
$\mathbf{4}$ & 33,5 & 20,0 & 0,0 & 36,0 & 90,0 & 4,0 \\
$\mathbf{5}$ & 21,0 & 20,0 & 0,0 & 23,5 & 90,0 & 4,0 \\
$\mathbf{6}$ & 31,0 & 55,0 & 0,0 & 33,5 & 90,0 & 1,0 \\
$\mathbf{7}$ & 31,0 & 55,0 & 2,0 & 33,5 & 90,0 & 4,0 \\
$\mathbf{8}$ & 23,5 & 20,0 & 0,0 & 26,0 & 90,0 & 4,0 \\
$\mathbf{9}$ & 16,0 & 20,0 & 2,0 & 21,0 & 90,0 & 4,0 \\
$\mathbf{1 0}$ & 31,0 & 55,0 & 1,0 & 33,5 & 90,0 & 2,0 \\
\hline
\end{tabular}




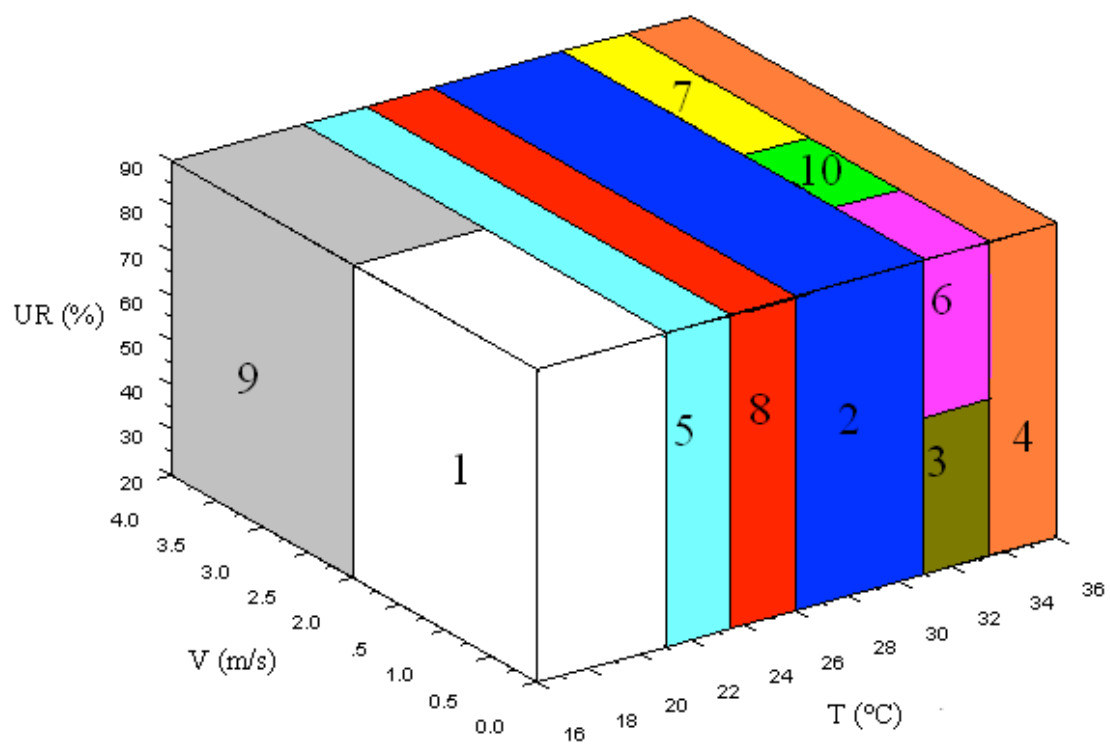

FIGURA 2. Superfície gerada segundo as partições realizadas no espaço de entrada.

Para construir um controlador neuro-fuzzy, baseada no algoritmo LOLIMOT, é necessário possuir a lista de cortes (inferior e superior) e os modelos lineares de acordo com cada partição. A rede neuro-fuzzy apresentou erro quadrático médio de treinamento e validação iguais a 0,20 e 0,24, respectivamente. Para avaliar a qualidade do ajuste realizado pela rede neuro-fuzzy, obteve-se os coeficientes de determinação $\left(\mathrm{R}^{2}\right)$ para o treinamento e validação, na qual foram iguais a 0,91 e 0,85 , respectivamente.

O desvio médio entre os resultados obtidos pela rede neuro-fuzzy e medidos experimentalmente, conforme as pesquisas realizadas por $[24,25,26,27]$, foi de $0,11{ }^{\circ} \mathrm{C}$, obtendo um $\mathrm{R}^{2}$ igual a 0,90 . Mesmo com um resíduo final de treinamento igual a 0,00516, considerado de certa maneira elevado, pode-se verificar por meio do desvio médio e coeficiente de determinação, que a rede neuro-fuzzy simula satisfatoriamente a temperatura retal de frangos de corte, sendo que um desvio médio igual a $0,11^{\circ} \mathrm{C}$ é aceitável, de acordo com especialistas, na etapa experimental em ambiência animal.

A Figura 3 apresenta simulações da $T R$ em função da $T$ e $U R$, considerando a $V$ constante em 0,6 e $2,4 \mathrm{~m} \mathrm{~s}^{-1}$. Com base nos resultados obtidos pela rede neuro-fuzzy, a $T R$ aumenta à medida que aumenta os valores da $T$. A $V$ influencia a $T R$ num ambiente com $T$ elevadas, estando assim de acordo com os resultados encontrados por [25]. Os resultados mostram que a $T R$ aumenta com o aumento da $U R$, porém em magnitude menor quando comparado ao aumento de $T$. 
A.

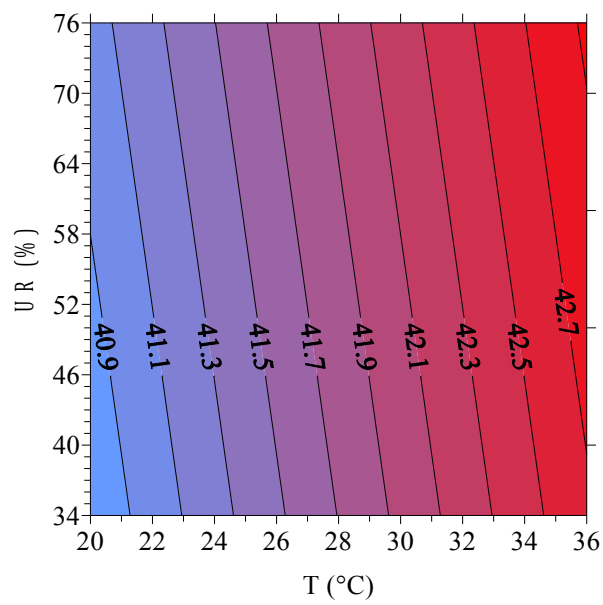

B.

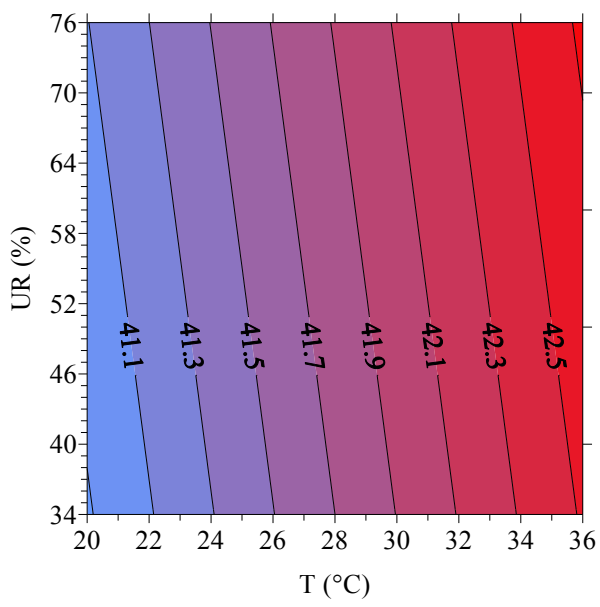

FIGURA 3. Estimativa da temperatura retal $\left(T R,{ }^{\circ} \mathrm{C}\right)$ pela rede neuro-fuzzy em função da temperatura do ar $(T)$, umidade relativa $(U R)$ e velocidades do ar de (A) $0,6 \mathrm{~m} \mathrm{~s}^{-1} \mathrm{e}$ (B) $2,4 \mathrm{~m} \mathrm{~s}^{-1}$.

A Figura 4 ilustra o comportamento da $T R$ simulada pela rede neuro-fuzzy com o auxílio do programa computacional 'SisTúnel', assim como a distribuição espacial da $T R$ (Figura 5) ao longo do galpão. Pelo comportamento da $T R$, verifica-se que o ambiente térmico sofreu alterações ao longo do galpão. Nos primeiros metros do galpão, na região que compreende a placa porosa umedecida, as aves estavam em conforto térmico e, à medida que o ar se desloca em direção à saída do galpão, o ambiente se torna mais desconfortável, estando de acordo com o verificado por diversos pesquisadores [28, 29]. 


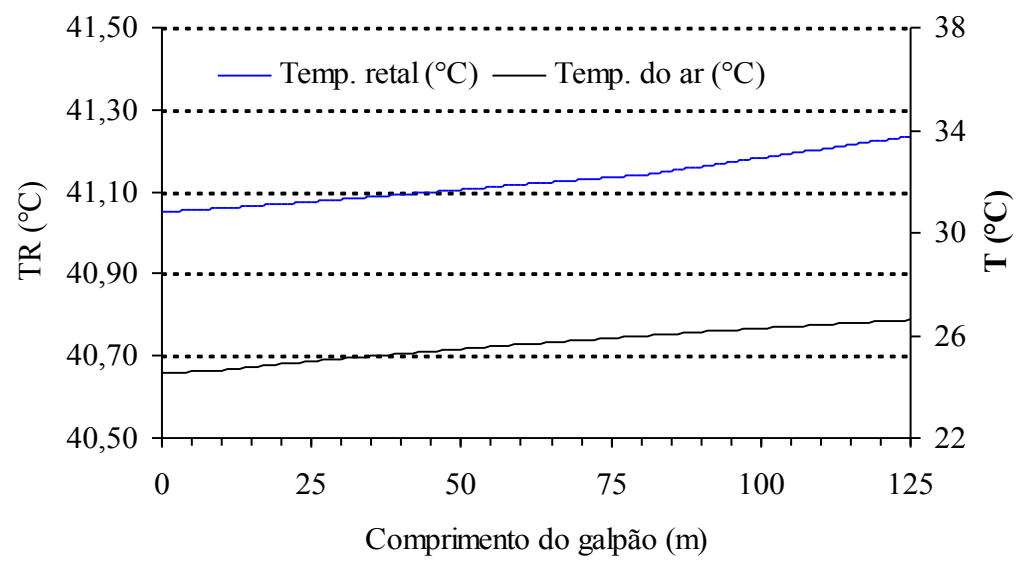

FIGURA 4. Representação do comportamento da $T R$ simulada pela rede neuro-fuzzy ao longo de um galpão climatizado.
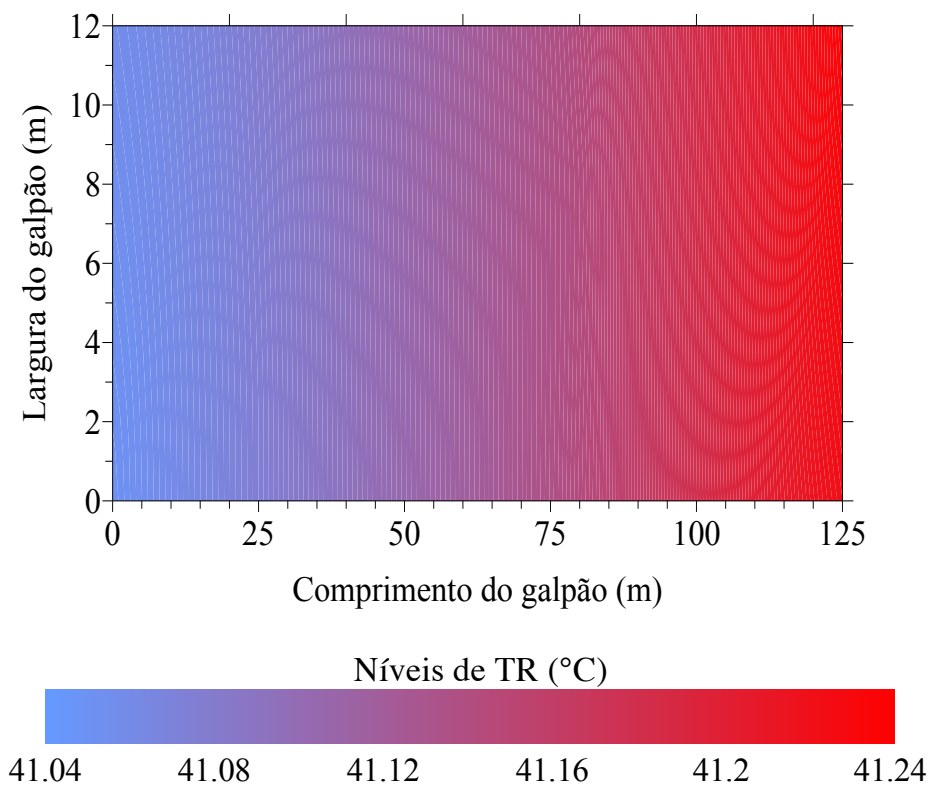

FIGURA 5. Distribuição espacial da $T R$ ao longo de um galpão climatizado. 


\section{Conclusões}

Por meio de um sistema inteligente híbrido, que utiliza a teoria dos conjuntos fuzzy e redes neurais artificiais, foi possível avaliar, satisfatoriamente, o ambiente de produção de frangos de corte, predizendo, com base nas variáveis ambientais, temperatura do ar $(T)$, umidade relativa $(U R)$ e velocidade do ar $(V)$, a temperatura retal do animal $(T R)$.

A rede neuro-fuzzy proposta, baseada no algoritmo LOLIMOT (Local Linear Model Tree), apresentou a capacidade de auto-aprendizado, sem a necessidade de conhecimento da relação entre as variáveis estudadas, e a potencialidade de trabalhar com as incertezas quanto ao ambiente de produção animal, com um mínimo processamento computacional.

\section{Agradecimentos}

Os autores agradecem à CAPES, CNPq e FAPEMIG pelo suporte a esta pesquisa.

\section{Referências}

[1] Silva, I, J. O. Ambiência na produção de aves em clima tropical. Jaboticabal: Editora Jaboticabal: SBEA, 2001. v. 1, 200 p.

[2] Curtis, S. E. Environmental management in animal agriculture. The Iowa State University: Ames, 1983. 410 p.

[3] Marchini, C. F. P.; Silva, P. L.; Nascimento, M. R. B. M.; Tavares, M. Frequência respiratória e temperatura cloacal em frangos de corte submetidos à temperatura ambiente cíclica elevada. Archives of Veterinary Science, Curitiba, v. 12, n. 1, p. 41-46, 2007.

[4] Moura, D. J. Ambiência na avicultura de corte. In: SILVA, I. J. O. Ambiência na produção de aves em clima tropical. Piracicaba: FUNEP, 2001. p. 75-149.

[5] Zadeh, L. A. Fuzzy Sets. Journal Information and Control, n. 8, p. 338-353, 1965.

[6] Klir, G. J.; Yuan, B. Fuzzy sets and fuzzy logic. New Jersey: Prentice Hall PTR, 1995. $574 \mathrm{p}$.

[7] Mendel, J. M. Fuzzy logic systems for engineering: A tutorial. Proceedings of IEEE, v. 83, n. 3, p. 345-377, Mar. 1995.

[8] Tsoukalas, L. H.; Uhrig, R. E. Fuzzy and Neural Approaches in Engineering. New York: John Wiley \& Sons, 1997. 600 p. 
[9] Lin, Y.; Cunningham III, G. A. A new approach to fuzzy-neural modelling, IEEE Transactions on Fuzzy Systems, v. 3, n. 2, p. 190-198, 1995.

[10] Figueiredo, M. F. Redes neurais nebulosas aplicadas em problemas de modelagem controle autônomo. 1997. 125 p. Tese (Doutorado em Engenharia Elétrica) - Universidade Estadual de Campinas, Campinas, SP.

[11] Mancini, F.; Chiao Yi, L.; Pignatari, S. S. N.; Roque, A. C. ; Pisa, I. T. Aplicação de redes neurais artificiais na classificação de padrões posturais em crianças respiradoras bucais e nasais. Revista de Informática Teórica e Aplicada, Porto Alegre, v. 14, n. 2, p. 91-107, 2007.

[12] Lopes, A. Z.; Ferreira, L.; Yanagi Junior, T.; Lacerda, W. S. Modeling productive performance of broiler chickens with artificial neural networks. In: CIGR International Conference of Agricultural Engineering - XXXVII Brazilian Congress of Agricultural Engineering - ILES VIII International Livestock Environment, 2008, Foz do Iguaçu. Anais... Foz do Iguaçu: SBEA, 2008. v. 1, p. 1-4.

[13] Schiassi, L.; Yanagi Junior, T.; Ferreira, L.; Damasceno, F. A.; Yanagi, S. N. M. Metodologia fuzzy aplicada à avaliação do aumento da temperatura corporal em frangos de corte. Engenharia na Agricultura, Viçosa, MG, v. 16, n. 2, p. 180-191, abr./jun. 2008.

[14] Ferreira, L.; Yanagi Junior, T.; Naas, I. A.; Lopes, M. A. Development of algorithm using fuzzy logic to predict estrus in dairy cows: Part I. Agricultural Engeneering International: The CIGR Ejournal, v. 9, p. 1-16, 2007.

[15] Ortega, N. R. S. Aplicação da teoria de conjuntos fuzzy a problemas da biomedicina. 2001. 152 p. Tese (Doutorado em Ciências) - Universidade de São Paulo, São Paulo, SP.

[16] Barros, L. C.; Bassanezi, R. C. Tópicos de lógica fuzzy e biomatemática. Campinas: IMECC-UNICAMP, 2006. 354 p.

[17] Castanho, M. J. P. Sistema neuro-fuzzy para prognóstico de câncer de próstata. Biomatemática, Campinas, n.17, p. 11-20, 2007.

[18] Fullér, R. Introduction in Neuro-fuzzy Systems. Berlin/ Heildelberg: Springer - Verlag, 2000. 289 p.

[19] Jang, J. S. R. ANFIS: Adaptive-Network-Based Fuzzy Inference System. IEEE Transactions on Systems, Man, and Cybernetics, New York, v. 23, n. 3, p. 665-685, 1993.

[20] Buckley, J. J.; Hayashi, Y. Neural networks for fuzzy systems. Fuzzy Sets and Systems, n. 71, p. $265-276,1995$. 
[21] SCILAB: Modeling and Control Tools - Lolimot 1.0. em: http://www.scilab.org/contrib/index_contrib.php?page=displayContribution\&fileID=997. Acessado em 04/07/2008.

[22] Nelles, O. Nonlinear system identification: from classical approaches to neural networks and fuzzy models. Berlin: Springer-Verlag, 2001. 785 p.

[23] Nelles, O.; Isermann, R. Basis function networks for interpolation of local linear models. In: Conference on Decision and Control, 1996, Kobe. Proceedings... Kobe: IEEE, 1996, p. 470-475.

[24] Sevegnani, K. B. Avaliação dos efeitos fisiológicos causados pela ventilação artificial em frangos de corte, em dispositivos de simulação climática. 2000. 96 p. Tese (Doutorado em Engenharia Agrícola) - Universidade de Campinas, Campinas, SP.

[25] Yahav, S.; Straschnow, A.; Luger, D.; Shinder, D.; Tanny, J.; Cohen, S. Ventilation, sensible heat loss, broiler energy, and water balance under harsh environmental conditions. Poultry Science, Champaign, v.83, n.2, p. 253-258, Feb. 2004.

[26] Souza, B. B.; Bertechini, A. G.; Teixeira, A. S.; Lima, J. A. F.; Conte, A. J.; Carvalho, C. H. O. Efeito do nível energético e da suplementação com cloretos de potássio e de amônia na dieta sobre as respostas fisiológicas e desempenho de frangos de corte no verão. Ciência e Agrotecnologia, Lavras, v.29, n.1, p. 185-192, jan./fev. 2005.

[27] Medeiros, C. M; Baêta, F. C.; Oliveira, R. F. M.; Tinôco, I. F. F.; Albino, L. F. T.; Cecon, P. R. Efeitos da temperatura, umidade relativa e velocidade do ar em frangos de corte. Engenharia na Agricultura, Viçosa, v.13, n.4, p. 277-286, out./nov. 2005.

[28] Carvalho, V. F. Modelagem e zoneamento do ambiente térmico para avaliar o desempenho de frangos de corte criados em galpões climatizados. 2006, 77 p. Dissertação (Mestrado em Engenharia Agrícola) - Universidade Federal de Lavras, Lavras, MG.

[29] Damasceno, F. A. Bem-estar do animal e do trabalhador em galpões avícolas climatizados. 2008. 220 p. Dissertação (Mestrado em Engenharia Agrícola) - Universidade Federal de Lavras, Lavras, MG. 